\title{
Organic Tomatoes: Combining Ability for fruit yield and Component Traits in Tomato (Solanum lycopersicum L.) under Mid Himalayan Region
}

\author{
Nisha Thakur*, Sanjay Chadha and Mayanglambam Bilashini Devi \\ Department of Vegetable Science and Floriculture, \\ CSK Himachal Pradesh Krishi Vishvavidyalaya, Palampur 176 062, India \\ *Corresponding author
}

\section{A B S T R A C T}

\section{Keywords}

\section{Solanum}

lycopersicum,

Organic, Standard

check, General

combining ability,

Specific combining

ability

\section{Article Info}

Accepted:

14 December 2018

Available Online:

10 January 2019
Combining ability effects were estimated for yield, yield components in a $8 \times 8$ diallel analysis excluding reciprocals. The variances for general combining ability (GCA) and specific combining ability (SCA) were highly significant indicating the presence of additive as well as non-additive gene effects in the traits studied. The relative magnitude of these variances indicated that additive gene effects were more prominent for all the characters. The tomato genotype Hawaii $7998\left(\mathrm{P}_{3}\right)$ proved to be the best general combiner for yield and its component traits followed by 12-1 ( $\left.\mathrm{P}_{5}\right)$ and BWR-5 $\left(\mathrm{P}_{6}\right)$.Cross combinations viz., Palam Pride $\times$ BWR-5 $\left(\mathrm{P}_{4} \times \mathrm{P}_{6}\right), 12-1 \times \mathrm{BWR}-5\left(\mathrm{P}_{5} \times\right.$ $\left.\mathrm{P}_{6}\right)$, Palam Pride $\times 12-1\left(\mathrm{P}_{4} \times \mathrm{P}_{5}\right)$, Hawaii $7998 \times 12-1\left(\mathrm{P}_{3} \times \mathrm{P}_{5}\right)$ and CLN 2123 A-1 red $\times$ Arka Abha $\left(\mathrm{P}_{2} \times \mathrm{P}_{8}\right)$ were the best five specific combinations for marketable yield per plant in pooled environment under organic farming conditions.

\section{Introduction}

Tomato (Solanum lycopersicum L.) is one of the most important vegetable crops grown throughout the world. It is used in fresh as well as processed food industries. Bacterial wilt has become a limiting factor for the commercial cultivation of tomato crop. Being safe and better in quality, the demand for organic tomatoes is increasing day by day. It is estimated that more than $95 \%$ of organic production is based on crop varieties that were bred for the conventional high-input sector. Recent studies have shown that such varieties lack important traits required under organic and low-input production conditions. This is primarily due to selection in conventional breeding programmes being carried out in the background of high inorganic fertilizer and crop protection inputs. Therefore high yielding organic input responsive varieties/hybrids with more pest tolerance/resistance are required. The hybrid cultivars in tomato have generated increased interest among the breeders due to possibility of combining a complex of valuable attributes 
in a genotype, viz. earliness, uniformity, high yield, resistance to diseases and strong adaptability to different environmental conditions. However in public sector there is still a dearth of F1 hybrids that have a complex of these valuable attributes. The systematic approach for developing F1 hybrids in any crop depends primarily on selection of desirable parents. The information obtained from general combining ability of parents and specific combining ability of crosses helps us to select suitable parents and cross combination respectively. An analysis of crosses produce by involving (n) lines in all possible combinations is known as a diallel analysis. This analysis is usually conducted to estimate the important genetic parameters; general combining ability (GCA), and specific combining ability (SCA) of the parents and crosses, respectively. Agroclimatic diversity acts as double-edged sword as in one hand it complicates the selection of suitable genotypes and on the other hand it gives information about the extreme environmental conditions which the genotype can withstand. Therefore, present investigation was planned to study the combining ability of some apparently superior genotypes for desirable horticultural traits across environment by involving bacterial wilt resistant parents under organic farming condition.

\section{Materials and Methods}

The tomato genotypes viz., CLN $2070\left(\mathrm{P}_{1}\right)$, CLN 2123 A-1 red $\left(\mathrm{P}_{2}\right)$, Hawaii $7998\left(\mathrm{P}_{3}\right)$, Palam Pride $\left(\mathrm{P}_{4}\right), 12-1\left(\mathrm{P}_{5}\right)$, BWR-5 $\left(\mathrm{P}_{6}\right)$, Arka Abha $\left(\mathrm{P}_{7}\right)$ and Arka Meghali $\left(\mathrm{P}_{8}\right)$ werecrossed in diallel fashion following Griffing (1956), model I, method II, at Model Organic Farm, Department of Organic Agriculture, COA, CSKHPKV, Palampur. Characteristics and source of the parents and checks involved in the study given in table 1. This farms is situated at $32^{\circ} 6^{\prime} \mathrm{N}$ latitude and $76^{\circ} 3^{\prime}$ E longitude at an altitude of $1290.8 \mathrm{~m}$ above the mean sea level. The parents and their resulting $28 \mathrm{~F} 1$ hybrids along with one standard check Avtar (7711) were evaluated in a randomized complete block design with three replications summer-rainy seasons. The seedlings were transplanted at the spacing of $75 \mathrm{~cm}$ between rows and $45 \mathrm{~cm}$ between plants. Recommended cultural practices were followed to raise a good crop. Data were collected for days to 50 per cent flowering, days to first harvest, gross yield per plant $(\mathrm{kg})$, marketable yield per plant $(\mathrm{kg})$, total number of fruits per plant, marketable fruits per plant, fruit weight $(\mathrm{g})$, fruit shape index, pericarp thickness $(\mathrm{mm})$, locules per fruit, plant height $(\mathrm{cm})$, harvest duration (days), total soluble solids (\%), ascorbic acid $(\mathrm{mg} / 100 \mathrm{~g})$ and titrable acidity (\%). The homogenized juice, obtained from 6 to 10 randomly chosen fruit for each genotype, was scored for soluble solid susing a manual Refractometer (A.O.A.C., 1970). The ascorbic acid contents and titrable acidity were estimated as described by Ranganna (1979). The diallel analysis was carried out as per Method 2 (parents plus one set of crosses and no reciprocal), Model I (fixed effect model) as described by Griffing (1956). The data was analysed for combining ability using gca and sca.

\section{Results and Discussion}

The analyses of variances for combining ability in 2012, 2013 and pooled over environments (Table 2) revealed that mean squares due to GCA were significant for all the traits studied in all the environments except harvest duration in 2013. Mean squares due to SCA were also found significant for all the traits studied except days to first harvest in all the environments, fruit shape index in 2012 and 2013, plant height in 2012 and TSS in 2013. Mean squares due to GCA $\times$ environment 
interaction were significant for all the traits studied except days to 50 per cent flowering, days to first harvest, fruit shape index, pericarp thickness and TSS, while mean square due to SCA $\times$ environment interaction were significant for all the traits studied except days to first harvest, fruit weight, fruit shape index, pericarp thickness, locules per fruit and TSS. Highly significant variation due to general combining ability as well as specific combining ability indicated the importance of additive as well as non-additive types of gene action for the expression of these traits. These findings are in close agreement with Farzane et al., (2013), Kumar et al., (2013), Saleem et al., (2013), Shankar et al., (2013) and Yadav et al., (2013).

\section{Estimation of general combining ability (GCA) effects}

Nature and magnitude of combining ability effects provide guideline in identifying the better parents and their utilization. The GCA effects of the parents (Table 3) revealed that none of the parent found to be good general combiner for all the characters. An overall appraisal of gca effects revealed that among parents $\mathrm{P}_{3}$ (Hawaii 7998) was found to be the best parent as it gave good general combining ability consistently in all the environments for maximum number of traits viz., days to 50 per cent flowering, gross yield per plant, total number of fruits per plant, marketable fruits per plant and plant height. $\mathrm{P}_{3}$ was also found good combiner for other traits studied viz., days to first harvest, marketable yield per plant, harvest duration, ascorbic acid and titrable acidity in pooled over environments. The second most desirable parent was observed to be $\mathrm{P}_{5}$ (12-1) which revealed significant desirable GCA effects for gross yield per plant, marketable yield per plant, fruit weight, fruit shape index, pericarp thickness and plant height in all the environments including total number of fruits per plant in 2012 and pooled environment and ascorbic acid in 2013 and pooled environment. $\mathrm{P}_{6}$ (BWR-5) was also a promising parent for inclusion in breeding programme as it revealed good general combing ability for marketable yield per plant, fruit weight and locules per fruit in all the environments, while it also exhibited significant desirable GCA effects for titrable acidity in 2012 and pooled environment.

\section{Estimates of specific combining ability (SCA) effects}

For days to 50 per cent flowering (Table 4), out of the 28 crosses studied, $\mathrm{P}_{4} \times \mathrm{P}_{7}$ (poor $\times$ good), $\mathrm{P}_{3} \times \mathrm{P}_{6}$ (good $\times$ average), $\mathrm{P}_{2} \times \mathrm{P}_{8}$ $\left(\right.$ good $\times$ good), $\mathrm{P}_{4} \times \mathrm{P}_{5}($ poor $\times$ poor $)$ and $\mathrm{P}_{4} \times$ $\mathrm{P}_{8}$ (poor $\times$ good) in 2012, $\mathrm{P}_{4} \times \mathrm{P}_{7}$ (average $\times$ good) and $\mathrm{P}_{1} \times \mathrm{P}_{7}$ (poor $\times$ good) in 2013 and $\mathrm{P}_{4} \times \mathrm{P}_{7}\left(\right.$ poor $\times$ good) and $\mathrm{P}_{2} \times \mathrm{P}_{8}(\operatorname{good} \times$ good) in pooled environment expressed significant negative SCA effects indicating their good specific combining ability. For days to first harvest SCA effects of the cross combinations in all the environments were not worked out due to non-significant mean square due to SCA. For gross yield per plant (Table 4), 12 cross combinations each in 2012 and 2013 and 13 crosses in pooled environment had positive significant SCA effects, thereby revealing their good specific combining ability. Out of these good specific combinations $\mathrm{P}_{1} \times \mathrm{P}_{3}, \mathrm{P}_{1} \times \mathrm{P}_{5}, \mathrm{P}_{2} \times \mathrm{P}_{6}, \mathrm{P}_{2} \times$ $\mathrm{P}_{7}, \mathrm{P}_{3} \times \mathrm{P}_{7}, \mathrm{P}_{4} \times \mathrm{P}_{6}, \mathrm{P}_{4} \times \mathrm{P}_{7}$ and $\mathrm{P}_{4} \times \mathrm{P}_{8}$ were common in all the environments. However, in order of preference in pooled environment $\mathrm{P}_{4}$ (average) $\times \mathrm{P}_{6}$ (average), $\mathrm{P}_{3}($ good $) \times \mathrm{P}_{7}$ (poor), $\mathrm{P}_{4}$ (average) $\times \mathrm{P}_{7}$ (poor), $\mathrm{P}_{4}$ (average) $\times \mathrm{P}_{8}$ (poor) and $\mathrm{P}_{1}($ good $) \times \mathrm{P}_{5}($ good $)$ were the most desirable specific combinations. For marketable yield per plant (Table 4), 10 cross combinations each in 2012 and 2013 and 11 cross combinations in pooled environment exhibited significant positive SCA effects (good specific combiners) for marketable 
yield per plant. The top five crosses were $\mathrm{P}_{4} \times$ $\mathrm{P}_{6}$ (average $\times$ good $), \mathrm{P}_{5} \times \mathrm{P}_{6}($ good $\times$ good $)$, $\mathrm{P}_{4} \times \mathrm{P}_{5}$ (average $\times$ good $), \mathrm{P}_{3} \times \mathrm{P}_{5}(\operatorname{good} \times$ good) and $\mathrm{P}_{2} \times \mathrm{P}_{8}$ (poor $\times$ poor) in pooled environment and were common in all the environments. For total number of fruits per plant (Table 4), Eight cross combinations each in 2012 and 2013 and 10 in pooled environment exhibited significant positive SCA effects indicating their good specific combining ability. Out of these cross $\mathrm{P}_{2} \times \mathrm{P}_{7}$ (average $\times$ poor $), \mathrm{P}_{3} \times \mathrm{P}_{6}($ good $\times$ poor $), \mathrm{P}_{3} \times$ $\mathrm{P}_{4}($ good $\times$ poor $), \mathrm{P}_{2} \times \mathrm{P}_{5}($ average $\times$ good $)$ and $\mathrm{P}_{5} \times \mathrm{P}_{8}(\operatorname{good} \times$ poor $)$ in pooled environment were the top five good specific combinations and $\mathrm{P}_{2} \times \mathrm{P}_{7}, \mathrm{P}_{3} \times \mathrm{P}_{6}$ and $\mathrm{P}_{2} \times \mathrm{P}_{5}$ were common in all the environments. Good specific combinations for marketable fruits per plant (Table 4) were $\mathrm{P}_{5} \times \mathrm{P}_{6}, \mathrm{P}_{4} \times \mathrm{P}_{6}, \mathrm{P}_{1} \times$ $\mathrm{P}_{7}, \mathrm{P}_{4} \times \mathrm{P}_{7}, \mathrm{P}_{2} \times \mathrm{P}_{8}, \mathrm{P}_{4} \times \mathrm{P}_{8}$ and $\mathrm{P}_{2} \times \mathrm{P}_{7}$ in 2012, $\mathrm{P}_{5} \times \mathrm{P}_{8}, \mathrm{P}_{3} \times \mathrm{P}_{5}, \mathrm{P}_{2} \times \mathrm{P}_{7}, \mathrm{P}_{3} \times \mathrm{P}_{4}$ and $\mathrm{P}_{6}$ $\times \mathrm{P}_{7}$ in 2013 and $\mathrm{P}_{5} \times \mathrm{P}_{6}, \mathrm{P}_{4} \times \mathrm{P}_{6}, \mathrm{P}_{3} \times \mathrm{P}_{5}, \mathrm{P}_{2}$ $\times \mathrm{P}_{7}, \mathrm{P}_{5} \times \mathrm{P}_{8}, \mathrm{P}_{3} \times \mathrm{P}_{4}, \mathrm{P}_{2} \times \mathrm{P}_{8}, \mathrm{P}_{4} \times \mathrm{P}_{7}, \mathrm{P}_{4} \times \mathrm{P}_{5}$ and $\mathrm{P}_{1} \times \mathrm{P}_{7}$ in pooled over environments. All the parents of these crosses were average or poor general combiners except $\mathrm{P}_{3}$ which was good general combiner in all the environments. Cross combination $\mathrm{P}_{2} \times \mathrm{P}_{7}$ was the common in all the environment for marketable fruits per plant. The computation of SCA effect for fruit weight (Table 5) indicated that the cross combinations $\mathrm{P}_{4} \times \mathrm{P}_{5}$ $($ good $\times$ good $), \mathrm{P}_{6} \times \mathrm{P}_{8}($ good $\times$ average $), \mathrm{P}_{1} \times$ $\mathrm{P}_{3}($ good $\times$ poor $), \mathrm{P}_{1} \times \mathrm{P}_{2}($ good $\times$ poor $), \mathrm{P}_{7} \times$ $\mathrm{P}_{8}$ (average $\times$ average), $\mathrm{P}_{2} \times \mathrm{P}_{3}$ (poor $\times$ poor) and $\mathrm{P}_{6} \times \mathrm{P}_{7}($ good $\times$ average $)$ in 2012, $\mathrm{P}_{5} \times \mathrm{P}_{6}$ $($ good $\times$ good $), \mathrm{P}_{4} \times \mathrm{P}_{6}($ good $\times$ good $), \mathrm{P}_{1} \times \mathrm{P}_{3}$ (average $\times$ poor), $\mathrm{P}_{1} \times \mathrm{P}_{2}$ (average $\times$ poor) and $\mathrm{P}_{2} \times \mathrm{P}_{3}$ (poor $\times$ poor) in 2013 and $\mathrm{P}_{1} \times \mathrm{P}_{3}$ (good $\times$ poor), $\mathrm{P}_{1} \times \mathrm{P}_{2}($ good $\times$ poor $), \mathrm{P}_{4} \times \mathrm{P}_{5}$ $($ good $\times$ good $), \mathrm{P}_{5} \times \mathrm{P}_{6}($ good $\times$ good $), \mathrm{P}_{2} \times \mathrm{P}_{3}$ (poor $\times$ poor), $\mathrm{P}_{7} \times \mathrm{P}_{8}$ (average $\times$ poor), $\mathrm{P}_{4} \times$ $\mathrm{P}_{6}(\operatorname{good} \times \operatorname{good})$ and $\mathrm{P}_{6} \times \mathrm{P}_{8}(\operatorname{good} \times$ poor $)$ in pooled environment showed significant positive SCA effects and the cross combinations viz., $\mathrm{P}_{1} \times \mathrm{P}_{2}, \mathrm{P}_{1} \times \mathrm{P}_{3}$ and $\mathrm{P}_{2} \times \mathrm{P}_{3}$ were common in all the environments. For fruit shape index (Table 5) SCA effects of the cross combinations in 2012 and 2013 were not worked out due to non-significant mean squares due to SCA. In pooled over environments, cross combinations viz., $\mathrm{P}_{4} \times \mathrm{P}_{7}$ (poor $\times$ poor), $\mathrm{P}_{6} \times \mathrm{P}_{8}$ (average $\times$ poor) and $\mathrm{P}_{3}$ $\times \mathrm{P}_{7}$ (average $\times$ poor) exhibited significant positive SCA effects indicating their good specific combining ability. For pericarp thickness (Table 5) in 2012, the crosses $\mathrm{P}_{3} \times$ $\mathrm{P}_{4}$ (poor $\times$ average), $\mathrm{P}_{4} \times \mathrm{P}_{7}$ (average $\times$ poor), $\mathrm{P}_{3} \times \mathrm{P}_{6}$ (poor $\times$ average) and $\mathrm{P}_{2} \times \mathrm{P}_{4}$ (average $\times$ average) in 2012, $\mathrm{P}_{4} \times \mathrm{P}_{7}$ (average $\times$ poor), $\mathrm{P}_{2} \times \mathrm{P}_{4}($ good $\times$ average $), \mathrm{P}_{3} \times \mathrm{P}_{4}$ (poor $\times$ average), $\mathrm{P}_{4} \times \mathrm{P}_{5}$ (average $\times$ good) and $\mathrm{P}_{3} \times \mathrm{P}_{6}$ (poor $\times$ average) in 2013 and $\mathrm{P}_{4} \times \mathrm{P}_{7}$ (average $\times$ poor), $\mathrm{P}_{3} \times \mathrm{P}_{4}$ (poor $\times$ average), $\mathrm{P}_{3} \times \mathrm{P}_{6}$ (poor $\times$ average), $\mathrm{P}_{2} \times \mathrm{P}_{4}$ (good $\times$ average), $\mathrm{P}_{5}$ $\times \mathrm{P}_{7}($ good $\times$ poor $), \mathrm{P}_{5} \times \mathrm{P}_{6}($ good $\times$ average $)$ and $\mathrm{P}_{1} \times \mathrm{P}_{8}$ (average $\times$ poor) in pooled environment revealed significant positive SCA effects indicating their good specific combining ability. The cross combinations $\mathrm{P}_{2}$ $\times \mathrm{P}_{4}, \mathrm{P}_{3} \times \mathrm{P}_{4}, \mathrm{P}_{3} \times \mathrm{P}_{6}$ and $\mathrm{P}_{4} \times \mathrm{P}_{7}$ were the common in all the environments for pericarp thickness. For locules per fruit (Table 6), cross combinations $\mathrm{P}_{7} \times \mathrm{P}_{8}($ good $\times$ good $)$ and $\mathrm{P}_{3} \times \mathrm{P}_{5}$ (poor $\times$ poor) in 2012 were good specific combinations, whereas 7 crosses viz., $\mathrm{P}_{1} \times \mathrm{P}_{2}(\operatorname{good} \times$ poor $), \mathrm{P}_{1} \times \mathrm{P}_{6}(\operatorname{good} \times$ good $)$, $\mathrm{P}_{3} \times \mathrm{P}_{4}$ (poor $\times$ average), $\mathrm{P}_{3} \times \mathrm{P}_{5}($ poor $\times$ poor), $\mathrm{P}_{4} \times \mathrm{P}_{5}$ (average $\times$ poor), $\mathrm{P}_{6} \times \mathrm{P}_{8}($ good $\times$ good $)$ and $\mathrm{P}_{7} \times \mathrm{P}_{8}(\operatorname{good} \times$ good $)$ in 2013 as well as in pooled environment exhibited significant positive SCA effects indicating their good specific combining ability. For plant height (Table 5), SCA effects of the cross combinations in 2012 were not worked out due to non-significant mean squares due to SCA. A total of 9 crosses each in 2012 and pooled environment exhibited significant positive SCA effects indicating their good specific combining ability and out of these cross combinations, $\mathrm{P}_{5}$ (good) $\times \mathrm{P}_{8}$ (poor), $\mathrm{P}_{5}$ $($ good $) \times \mathrm{P}_{6}($ poor $), \mathrm{P}_{3}($ good $) \times \mathrm{P}_{6}($ poor $), \mathrm{P}_{5}$ 
$($ good $) \times \mathrm{P}_{7}($ poor $)$ and $\mathrm{P}_{3}($ good $) \times \mathrm{P}_{7}($ poor $)$ in pooled environment were the top five good specific combinations. For harvest duration (Table 6) the perusal of SCA effects revealed that the crosses viz., $\mathrm{P}_{3} \times \mathrm{P}_{8}, \mathrm{P}_{4} \times \mathrm{P}_{7}, \mathrm{P}_{2} \times \mathrm{P}_{5}$, $\mathrm{P}_{6} \times \mathrm{P}_{8}, \mathrm{P}_{1} \times \mathrm{P}_{4}$ and $\mathrm{P}_{1} \times \mathrm{P}_{2}$ in 2012, $\mathrm{P}_{1} \times \mathrm{P}_{3}$, $\mathrm{P}_{1} \times \mathrm{P}_{7}$ and $\mathrm{P}_{4} \times \mathrm{P}_{7}$ in 2013 and $\mathrm{P}_{4} \times \mathrm{P}_{7}, \mathrm{P}_{3} \times$ $\mathrm{P}_{8}, \mathrm{P}_{2} \times \mathrm{P}_{5}, \mathrm{P}_{1} \times \mathrm{P}_{3}$ and $\mathrm{P}_{6} \times \mathrm{P}_{8}$ in pooled environment had significant positive SCA effects indicating their good specific combinations. All the parents of these crosses were average or poor general combiners except $\mathrm{P}_{3}$ which was good general combiner in pooled environment.

The cross combination $\mathrm{P}_{4} \times \mathrm{P}_{7}$ was common in all the environments. For total soluble solids (Table 6), SCA effects of the cross combinations in 2013 was not worked out due to non-significant mean squares due to SCA. Significant positive SCA effects were observed for the cross combinations $\mathrm{P}_{7} \times \mathrm{P}_{8}$, $\mathrm{P}_{5} \times \mathrm{P}_{8}, \mathrm{P}_{1} \times \mathrm{P}_{3}, \mathrm{P}_{1} \times \mathrm{P}_{5}$ and $\mathrm{P}_{3} \times \mathrm{P}_{7}$ in 2012 and they had average general combiners as their parents except $\mathrm{P}_{1}$ which was good general combiner. In pooled environment, $\mathrm{P}_{7}$ $\times \mathrm{P}_{8}$ (poor $\times$ average), $\mathrm{P}_{3} \times \mathrm{P}_{7}$ (average $\times$ poor), $\mathrm{P}_{1} \times \mathrm{P}_{3}$ (good $\times$ average), $\mathrm{P}_{5} \times \mathrm{P}_{8}$ (average $\times$ average), $\mathrm{P}_{2} \times \mathrm{P}_{6}($ good $\times$ poor $)$ and $\mathrm{P}_{6} \times \mathrm{P}_{7}$ (poor $\times$ poor) exhibited significant positive SCA effects indicating their good specific combining ability. For ascorbic acid (Table 6), a total of 10 crosses each in 2012 and pooled environment and 7 crosses in 2013 exhibited significant positive SCA effects indicating their good specific combining ability.

Out of these cross combinations $\mathrm{P}_{1}$ (average) $\times \mathrm{P}_{2}$ (poor), $\mathrm{P}_{4}($ good $) \times \mathrm{P}_{8}($ poor $), \mathrm{P}_{5}($ good $) \times$ $\mathrm{P}_{7}$ (poor), $\mathrm{P}_{1}$ (average) $\times \mathrm{P}_{6}$ (average) and $\mathrm{P}_{6}$ (average) $\times \mathrm{P}_{7}$ (poor) in pooled environment were the top five good specific combinations. Cross combinations $\mathrm{P}_{1} \times \mathrm{P}_{2}, \mathrm{P}_{4} \times \mathrm{P}_{8}$ and $\mathrm{P}_{6} \times$ $\mathrm{P}_{7}$ were common in all the environments. For titrable acidity (Table 6), 10 crosses each in 2012 and 2013 and 17 crosses in pooled environment exhibited significant positive SCA effects indicating their good specific combining ability. In order of preference, $\mathrm{P}_{6} \times$ $\mathrm{P}_{7}($ good $\times$ good $), \mathrm{P}_{6} \times \mathrm{P}_{8}($ good $\times$ good $), \mathrm{P}_{1} \times$ $\mathrm{P}_{4}$ (poor $\times$ poor), $\mathrm{P}_{2} \times \mathrm{P}_{4}$ (poor $\times$ poor) and $\mathrm{P}_{3}$ $\times \mathrm{P}_{7}($ good $\times$ good $)$ in pooled environment were the most desirable specific combinations. The cross combinations viz., $\mathrm{P}_{1}$ $\times \mathrm{P}_{4}, \mathrm{P}_{2} \times \mathrm{P}_{4}, \mathrm{P}_{3} \times \mathrm{P}_{7}, \mathrm{P}_{3} \times \mathrm{P}_{8}, \mathrm{P}_{6} \times \mathrm{P}_{7}$ and $\mathrm{P}_{6}$ $\times \quad \mathrm{P}_{8}$ were common in all the environments.Our results are in close conformity with the findings of Rattan et al., (2008), Singh et al., (2010) and Singh and Asati (2011). Our results are in close conformity with the findings of Joshi et al., (2005), Pandey et al., (2006), Sharma et al., (2007), Chishti et al., (2008), Ahmad et al., (2009), Sharma and Sharma (2010), Singh et al., (2010), Dhaliwal and Cheema (2011), Singh and Asati (2011), Kumar et al., (2013), Saleem et al., (2013), Shankar et al., (2013) and Yadav et al., (2013).

Majority of the cross combinations exhibiting desirable SCA effects, had one of the parents atleast as good or average general combiner. Similar views have also been expressed by earlier researchers, Sharma and Sharma (2010), Singh and Asati (2011), Kumar et al., (2013), Saleem et al., (2013) and Yadav et al., (2013). However, certain crosses also revealed good SCA effects although the parents of these crosses had poor $\times$ poor or average $\times$ poor GCA effects. This might be due to the origin of parental lines used in the present study from the diverse genetic background thereby exhibiting high SCA effects. The poor $\times$ poor crosses may perform better than $\operatorname{good} \times \operatorname{good}$ and $\operatorname{good} \times$ poor combinations because of complimentary gene action. These findings corroborate the observations of Dhaliwal and Cheema (2011), Kumar et al., (2013b) and Shankar et al., (2013), who have also reported that the superior hybrids need not necessarily have parents showing high GCA effects only. 
Table.1 Characteristics of the parents and checks involved in the study

\begin{tabular}{|c|c|c|c|c|c|}
\hline Genotypes & $\begin{array}{l}\text { Code } \\
\text { No. }\end{array}$ & Sources & Growth habit & $\begin{array}{l}\text { Bacterial } \\
\text { wilt }\end{array}$ & $\begin{array}{l}\text { Fruit shape, } \\
\text { pedicel area and } \\
\text { colour }\end{array}$ \\
\hline CLN 2070 & $\mathrm{P}_{1}$ & $\begin{array}{l}\text { AVRDC/ CSK } \\
\text { HPKV }\end{array}$ & $\begin{array}{l}\text { Semi } \\
\text { determinate }\end{array}$ & Resistant & $\begin{array}{l}\text { Slightly flattened, } \\
\text { medium, orange } \\
\text { red colour }\end{array}$ \\
\hline $\begin{array}{l}\text { CLN } 2123 \text { A- } \\
1 \text { (red) }\end{array}$ & $\mathrm{P}_{2}$ & $\begin{array}{l}\text { AVRDC/CSK } \\
\text { HPKV }\end{array}$ & Determinate & Resistant & $\begin{array}{l}\text { Ovoid, shallow, } \\
\text { deep red }\end{array}$ \\
\hline Hawaii 7998 & $\mathrm{P}_{3}$ & $\begin{array}{l}\text { AVRDC/ } \\
\text { CSKHPKV }\end{array}$ & Indeterminate & Resistant & $\begin{array}{l}\text { Circular, shallow, } \\
\text { red }\end{array}$ \\
\hline Palam Pride & $\mathrm{P}_{4}$ & $\begin{array}{l}\text { AVRDC/CSK } \\
\text { HPKV }\end{array}$ & Indeterminate & Resistant & $\begin{array}{l}\text { Heart shaped, } \\
\text { shallow, red }\end{array}$ \\
\hline $12-1$ & $\mathrm{P}_{5}$ & CSKHPKV & Indeterminate & Resistant & $\begin{array}{l}\text { Obovoid, shallow, } \\
\text { red }\end{array}$ \\
\hline BWR-5 & $\mathrm{P}_{6}$ & IIHR/CSKHPKV & Determinate & & $\begin{array}{l}\text { Rectangular, deep, } \\
\text { orange red }\end{array}$ \\
\hline Arka Abha & $\mathrm{P}_{7}$ & IIHR & $\begin{array}{l}\text { Semi- } \\
\text { determinate }\end{array}$ & $\begin{array}{l}\text { Moderate } \\
\text { resistant }\end{array}$ & $\begin{array}{l}\text { Flattened, } \\
\text { medium, red }\end{array}$ \\
\hline $\begin{array}{l}\text { Arka } \\
\text { Meghali }\end{array}$ & $\mathrm{P}_{8}$ & IIHR & $\begin{array}{l}\text { Semi- } \\
\text { determinate }\end{array}$ & $\begin{array}{l}\text { Moderate } \\
\text { susceptible }\end{array}$ & $\begin{array}{l}\text { Flattened, } \\
\text { medium, red }\end{array}$ \\
\hline \multicolumn{6}{|c|}{ Standard check } \\
\hline Avtar (7711) & $\mathrm{SC}$ & Nunhems & Indeterminate & Resistant & $\begin{array}{l}\text { Obovoid, shallow, } \\
\text { red }\end{array}$ \\
\hline \multicolumn{6}{|c|}{ Susceptible check } \\
\hline Roma & & IARI/CSKHPKV & Determinate & Susceptible & $\begin{array}{l}\text { Cylindrical, } \\
\text { absent, red }\end{array}$ \\
\hline Marglobe & & IARI/CSKHPKV & Indeterminate & Susceptible & $\begin{array}{l}\text { Round, medium, } \\
\text { red }\end{array}$ \\
\hline
\end{tabular}


Table.2 Analyses of variances for combining ability for different traits in tomato during 2012, 2013 and pooled over environments under organic conditions

\begin{tabular}{|c|c|c|c|c|c|c|c|c|}
\hline $\begin{array}{l}\text { Source } \\
\text { of variation }\end{array}$ & $\rightarrow$ & GCA & SCA & Error & $\begin{array}{l}\text { Environme } \\
\text { nt }\end{array}$ & $\begin{array}{l}\text { GCA } \times \\
\text { Environm } \\
\text { ent }\end{array}$ & $\begin{array}{l}\text { SCA } \times \\
\text { Environmen } \\
t\end{array}$ & $\begin{array}{l}\text { Pooled } \\
\text { error }\end{array}$ \\
\hline Traits & $\mathrm{df} \rightarrow$ & 7 & 28 & 70 & 1 & 7 & 28 & 140 \\
\hline \multirow{3}{*}{$\begin{array}{l}\text { Days to } 50 \text { per } \\
\text { cent flowering }\end{array}$} & 2012 & $36.921^{*}$ & $3.370^{*}$ & 0.714 & - & - & - & - \\
\hline & 2013 & $29.267^{*}$ & $4.972^{*}$ & 3.711 & - & - & - & - \\
\hline & Pooled & $64.839^{*}$ & $4.127^{*}$ & - & $399.824^{*}$ & 1.350 & $4.214^{*}$ & 2.213 \\
\hline \multirow{3}{*}{$\begin{array}{l}\text { Days to first } \\
\text { harvest }\end{array}$} & 2012 & $50.270^{*}$ & 9.620 & 7.646 & - & - & - & - \\
\hline & 2013 & $31.032^{*}$ & 6.536 & 5.190 & - & - & - & - \\
\hline & Pooled & $73.257^{*}$ & 8.583 & - & $254.408^{*}$ & 8.045 & 7.573 & 6.418 \\
\hline \multirow{3}{*}{$\begin{array}{l}\text { Gross } \\
\text { yield/plant }\end{array}$} & 2012 & $0.091^{*}$ & $0.064^{*}$ & 0.008 & - & - & - & - \\
\hline & 2013 & $0.494^{*}$ & $0.171^{*}$ & 0.010 & - & - & - & - \\
\hline & Pooled & $0.480^{*}$ & $0.186^{*}$ & - & 0.0004 & $0.104^{*}$ & $0.048^{*}$ & 0.009 \\
\hline \multirow{3}{*}{$\begin{array}{l}\text { Marketable } \\
\text { yield/plant }\end{array}$} & 2012 & $0.139^{*}$ & $0.066^{*}$ & 0.007 & - & - & - & - \\
\hline & 2013 & $0.055^{*}$ & $0.036^{*}$ & 0.006 & - & - & - & - \\
\hline & Pooled & $0.159^{*}$ & $0.077^{*}$ & - & $1.292^{*}$ & $0.035^{*}$ & $0.025^{*}$ & 0.006 \\
\hline \multirow{3}{*}{$\begin{array}{l}\text { Total number } \\
\text { of fruits/plant }\end{array}$} & 2012 & $316.704^{*}$ & $30.621^{*}$ & 5.160 & - & - & - & - \\
\hline & 2013 & $234.112^{*}$ & $16.115^{*}$ & 1.254 & - & - & - & - \\
\hline & Pooled & $529.201^{*}$ & $29.123^{*}$ & - & $189.088^{*}$ & $21.614^{*}$ & $17.613^{*}$ & 3.207 \\
\hline \multirow{3}{*}{$\begin{array}{l}\text { Marketable } \\
\text { fruits/plant }\end{array}$} & 2012 & $146.577^{*}$ & $19.490^{*}$ & 2.624 & - & - & - & - \\
\hline & 2013 & $48.153^{*}$ & $9.102^{*}$ & 1.239 & - & - & - & - \\
\hline & Pooled & $165.445^{*}$ & $14.912^{*}$ & - & $350.220^{*}$ & $29.285^{*}$ & $13.681^{*}$ & 1.932 \\
\hline \multirow[t]{3}{*}{ Fruit weight } & 2012 & $235.022^{*}$ & $24.157^{*}$ & 3.730 & - & - & - & - \\
\hline & 2013 & $185.392^{*}$ & $21.728^{*}$ & 5.704 & - & - & - & - \\
\hline & Pooled & $409.160^{*}$ & $41.116^{*}$ & - & $130.876^{*}$ & $11.254^{*}$ & 4.770 & 4.717 \\
\hline
\end{tabular}


Int.J.Curr.Microbiol.App.Sci (2019) 8(1): 2099-2112

\begin{tabular}{|c|c|c|c|c|c|c|c|c|}
\hline \multirow{3}{*}{$\begin{array}{l}\text { Fruit shape } \\
\text { index }\end{array}$} & 2012 & $0.028^{*}$ & 0.002 & 0.001 & - & - & - & - \\
\hline & 2013 & $0.024^{*}$ & 0.002 & 0.001 & - & - & - & - \\
\hline & Pooled & $0.052^{*}$ & $0.003^{*}$ & - & 0.001 & 0.001 & 0.0004 & 0.001 \\
\hline \multirow{3}{*}{$\begin{array}{l}\text { Pericarp } \\
\text { thickness }\end{array}$} & 2012 & $0.478^{*}$ & $0.208^{*}$ & 0.060 & - & - & - & - \\
\hline & 2013 & $0.492^{*}$ & $0.202^{*}$ & 0.048 & - & - & - & - \\
\hline & Pooled & $0.932^{*}$ & $0.384^{*}$ & - & $0.277^{*}$ & 0.037 & 0.027 & 0.054 \\
\hline \multirow{3}{*}{$\begin{array}{l}\text { Locules per } \\
\text { fruit }\end{array}$} & 2012 & $1.958^{*}$ & $0.143^{*}$ & 0.047 & - & - & - & - \\
\hline & 2013 & $2.269^{*}$ & $0.172^{*}$ & 0.025 & - & - & - & - \\
\hline & Pooled & $4.141^{*}$ & $0.281^{*}$ & & 0.061 & $0.086^{*}$ & 0.034 & 0.036 \\
\hline \multirow[t]{3}{*}{ Plant height } & 2012 & $771.769^{*}$ & 41.905 & 28.175 & - & - & - & - \\
\hline & 2013 & $2624.965^{*}$ & $239.146^{*}$ & 26.482 & - & - & - & - \\
\hline & Pooled & $3034.157^{*}$ & $203.758^{*}$ & - & $353.975^{*}$ & $362.578^{*}$ & $77.294^{*}$ & 27.328 \\
\hline \multirow{3}{*}{$\begin{array}{l}\text { Harvest } \\
\text { duration }\end{array}$} & 2012 & $201.244^{*}$ & $47.152^{*}$ & 10.922 & - & - & - & - \\
\hline & 2013 & 9.531 & $25.414^{*}$ & 6.887 & - & - & - & - \\
\hline & Pooled & $110.647^{*}$ & $24.593^{*}$ & - & $296.085^{*}$ & $100.128^{*}$ & $47.973^{*}$ & 8.904 \\
\hline \multirow[t]{3}{*}{ TSS } & 2012 & $0.375^{*}$ & $0.108^{*}$ & 0.038 & - & - & - & - \\
\hline & 2013 & $0.277^{*}$ & 0.079 & 0.065 & - & - & - & - \\
\hline & Pooled & $0.583^{*}$ & $0.152^{*}$ & - & $0.205^{*}$ & 0.069 & 0.035 & 0.051 \\
\hline \multirow[t]{3}{*}{ Ascorbic acid } & 2012 & $2.918^{*}$ & $12.391^{*}$ & 0.757 & - & - & - & - \\
\hline & 2013 & $16.511^{*}$ & $10.122^{*}$ & 0.798 & - & - & - & - \\
\hline & Pooled & $10.736^{*}$ & $13.570^{*}$ & - & 0.880 & $8.693^{*}$ & $8.943^{*}$ & 0.777 \\
\hline \multirow{3}{*}{$\begin{array}{l}\text { Titrable } \\
\text { acidity }\end{array}$} & 2012 & $0.023^{*}$ & $0.007^{*}$ & 0.0003 & - & - & - & - \\
\hline & 2013 & $0.010^{*}$ & $0.007^{*}$ & 0.0004 & - & - & - & - \\
\hline & Pooled & $0.028^{*}$ & $0.010^{*}$ & - & $0.066^{*}$ & $0.005^{*}$ & $0.003^{*}$ & 0.0004 \\
\hline
\end{tabular}

${ }^{*}$ Significant at $5 \%$ level of significance 
Table.3 Estimates of general combining ability effects of parents for different traits in tomato during 2012, 2013 and pooled over environments under organic conditions

\begin{tabular}{|c|c|c|c|c|c|c|c|c|c|c|c|c|c|}
\hline Traits & lines $\rightarrow$ & 1 & 2 & 3 & 4 & 5 & 6 & 7 & 8 & $\mathrm{SE}($ gi) \pm & $\begin{array}{l}\text { SE(gi-gj) } \\
\pm\end{array}$ & CD (gi) & $\begin{array}{l}\text { CD (gi- } \\
\text { gj) }\end{array}$ \\
\hline \multirow{3}{*}{$\begin{array}{l}\text { Days to } 50 \\
\text { per cent } \\
\text { flowering }\end{array}$} & 2012 & $2.22^{*}$ & $-1.02^{*}$ & $-2.52^{*}$ & $1.08^{*}$ & $2.95^{*}$ & -0.12 & $-1.68^{*}$ & $-0.92^{*}$ & 0.25 & 0.38 & 0.50 & 0.75 \\
\hline & 2013 & $2.01^{*}$ & $-1.46^{*}$ & $-1.43^{*}$ & 0.84 & $2.91^{*}$ & -0.46 & $-1.23^{*}$ & $-1.19^{*}$ & 0.57 & 0.86 & 1.14 & 1.72 \\
\hline & Pooled & $2.11^{*}$ & $-1.24^{*}$ & $-1.97^{*}$ & $0.96^{*}$ & $2.93^{*}$ & -0.29 & $-1.45^{*}$ & $-1.05^{*}$ & 0.31 & 0.47 & 0.62 & 0.94 \\
\hline \multirow{3}{*}{$\begin{array}{l}\text { Days to first } \\
\text { harvest }\end{array}$} & 2012 & $2.88^{*}$ & $-1.88^{*}$ & $-3.02^{*}$ & 0.08 & $3.52^{*}$ & -0.08 & -1.35 & -0.15 & 0.82 & 1.24 & 1.63 & 2.46 \\
\hline & 2013 & $2.33^{*}$ & -1.17 & -0.83 & $1.37^{*}$ & $2.20^{*}$ & -0.27 & $-2.47^{*}$ & -1.17 & 0.67 & 1.02 & 1.34 & 2.03 \\
\hline & Pooled & $2.61^{*}$ & $-1.52^{*}$ & $-1.92^{*}$ & 0.73 & $2.86^{*}$ & -0.17 & $-1.91^{*}$ & -0.66 & 0.53 & 0.80 & 1.06 & 1.60 \\
\hline \multirow{3}{*}{$\begin{array}{l}\text { Gross } \\
\text { yield/plant }\end{array}$} & 2012 & $0.09^{*}$ & $-0.09^{*}$ & $0.15^{*}$ & -0.02 & $0.09^{*}$ & -0.04 & $-0.08^{*}$ & $-0.10^{*}$ & 0.03 & 0.04 & 0.05 & 0.08 \\
\hline & 2013 & $0.06^{*}$ & $-0.27^{*}$ & $0.44^{*}$ & 0.00 & $0.08^{*}$ & 0.04 & $-0.15^{*}$ & $-0.21^{*}$ & 0.03 & 0.04 & 0.06 & 0.09 \\
\hline & Pooled & $0.08^{*}$ & $-0.18^{*}$ & $0.30^{*}$ & -0.01 & $0.08^{*}$ & 0.00 & $-0.11^{*}$ & $-0.15^{*}$ & 0.02 & 0.03 & 0.04 & 0.06 \\
\hline \multirow{3}{*}{$\begin{array}{l}\text { Marketable } \\
\text { yield/plant }\end{array}$} & 2012 & 0.01 & $-0.11^{*}$ & $0.14^{*}$ & -0.04 & $0.13^{*}$ & $0.12^{*}$ & $-0.12^{*}$ & $-0.13^{*}$ & 0.02 & 0.04 & 0.05 & 0.07 \\
\hline & 2013 & $-0.10^{*}$ & $-0.06^{*}$ & 0.03 & 0.01 & $0.12^{*}$ & $0.07^{*}$ & $-0.06^{*}$ & -0.02 & 0.02 & 0.03 & 0.04 & 0.07 \\
\hline & Pooled & $-0.04^{*}$ & $-0.09^{*}$ & $0.09^{*}$ & -0.01 & $0.12^{*}$ & $0.10^{*}$ & $-0.09^{*}$ & $-0.07^{*}$ & 0.02 & 0.02 & 0.03 & 0.05 \\
\hline \multirow{3}{*}{$\begin{array}{l}\text { Total } \\
\text { number of } \\
\text { fruits/plant }\end{array}$} & 2012 & $-1.69^{*}$ & -0.73 & $13.15^{*}$ & $-2.79^{*}$ & $1.41^{*}$ & -1.06 & $-4.35^{*}$ & $-3.94^{*}$ & 0.67 & 1.02 & 1.34 & 2.02 \\
\hline & 2013 & $-4.88^{*}$ & -0.25 & $11.34^{*}$ & $-1.11^{*}$ & 0.28 & $-1.90^{*}$ & $-2.24^{*}$ & $-1.24^{*}$ & 0.33 & 0.50 & 0.66 & 1.00 \\
\hline & Pooled & $-3.29^{*}$ & -0.49 & $12.24^{*}$ & $-1.95^{*}$ & $0.85^{*}$ & $-1.48^{*}$ & $-3.29^{*}$ & $-2.59^{*}$ & 0.37 & 0.57 & 0.75 & 1.13 \\
\hline \multirow{3}{*}{$\begin{array}{l}\text { Marketable } \\
\text { fruits/plant }\end{array}$} & 2012 & $-0.98^{*}$ & $-1.09^{*}$ & $8.91^{*}$ & $-2.00^{*}$ & 0.71 & 0.19 & $-3.14^{*}$ & $-2.59^{*}$ & 0.48 & 0.72 & 0.95 & 1.44 \\
\hline & 2013 & $-2.84^{*}$ & -0.16 & $4.80^{*}$ & -0.23 & -0.01 & $-1.11^{*}$ & $-1.01^{*}$ & 0.56 & 0.33 & 0.50 & 0.66 & 0.99 \\
\hline & Pooled & $-1.91^{*}$ & $-0.63^{*}$ & $6.85^{*}$ & $-1.12^{*}$ & 0.35 & -0.46 & $-2.08^{*}$ & $-1.02^{*}$ & 0.29 & 0.44 & 0.58 & 0.88 \\
\hline \multirow{3}{*}{$\begin{array}{l}\text { Fruit } \\
\text { weight }\end{array}$} & 2012 & $1.53^{*}$ & $-3.52^{*}$ & $-9.51^{*}$ & $1.16^{*}$ & $4.60^{*}$ & $5.91^{*}$ & 0.89 & -1.06 & 0.57 & 0.86 & 1.14 & 1.72 \\
\hline & 2013 & 1.09 & $-2.22^{*}$ & $-7.51^{*}$ & $2.57^{*}$ & $4.78^{*}$ & $5.32^{*}$ & -1.19 & $-2.85^{*}$ & 0.71 & 1.07 & 1.41 & 2.13 \\
\hline & Pooled & $1.31^{*}$ & $-2.87^{*}$ & $-8.51^{*}$ & $1.87^{*}$ & $4.69^{*}$ & $5.62^{*}$ & -0.15 & $-1.96^{*}$ & 0.45 & 0.69 & 0.91 & 1.37 \\
\hline
\end{tabular}


Int.J.Curr.Microbiol.App.Sci (2019) 8(1): 2099-2112

\begin{tabular}{|c|c|c|c|c|c|c|c|c|c|c|c|c|c|}
\hline \multirow{3}{*}{$\begin{array}{l}\text { Fruit shape } \\
\text { index }\end{array}$} & 2012 & 0.01 & $0.08^{*}$ & -0.01 & $-0.02^{*}$ & $0.06^{*}$ & 0.01 & $-0.07^{*}$ & $-0.06^{*}$ & 0.01 & 0.02 & 0.02 & 0.03 \\
\hline & 2013 & -0.01 & $0.08^{*}$ & 0.00 & $-0.02^{*}$ & $0.05^{*}$ & $0.02^{*}$ & $-0.06^{*}$ & $-0.06^{*}$ & 0.01 & 0.02 & 0.02 & 0.03 \\
\hline & Pooled & 0.001 & $0.08^{*}$ & 0.00 & $-0.02^{*}$ & $0.05^{*}$ & 0.01 & $-0.06^{*}$ & $-0.06^{*}$ & 0.01 & 0.01 & 0.01 & 0.02 \\
\hline \multirow{3}{*}{$\begin{array}{l}\text { Pericarp } \\
\text { thickness }\end{array}$} & 2012 & $0.18^{*}$ & 0.11 & $-0.17^{*}$ & 0.12 & $0.29^{*}$ & 0.03 & $-0.24^{*}$ & $-0.33^{*}$ & 0.07 & 0.11 & 0.14 & 0.22 \\
\hline & 2013 & $0.18^{*}$ & $0.18^{*}$ & $-0.27^{*}$ & 0.04 & $0.34^{*}$ & -0.08 & $-0.15^{*}$ & $-0.24^{*}$ & 0.07 & 0.10 & 0.13 & 0.20 \\
\hline & Pooled & $0.18^{*}$ & $0.15^{*}$ & $-0.22^{*}$ & 0.08 & $0.32^{*}$ & -0.02 & $-0.19^{*}$ & $-0.29^{*}$ & 0.05 & 0.07 & 0.10 & 0.15 \\
\hline \multirow{3}{*}{$\begin{array}{l}\text { Locules per } \\
\text { fruit }\end{array}$} & 2012 & 0.12 & $-0.66^{*}$ & $-0.57^{*}$ & 0.05 & $-0.21^{*}$ & $0.37^{*}$ & $0.43^{*}$ & $0.48^{*}$ & 0.06 & 0.10 & 0.13 & 0.19 \\
\hline & 2013 & $0.16^{*}$ & $-0.65^{*}$ & $-0.46^{*}$ & 0.02 & $-0.49^{*}$ & $0.54^{*}$ & $0.46^{*}$ & $0.43^{*}$ & 0.05 & 0.07 & 0.09 & 0.14 \\
\hline & Pooled & $0.14^{*}$ & $-0.66^{*}$ & $-0.51^{*}$ & 0.03 & $-0.35^{*}$ & $0.46^{*}$ & $0.44^{*}$ & $0.45^{*}$ & 0.04 & 0.06 & 0.08 & 0.12 \\
\hline \multirow[t]{3}{*}{ Plant height } & 2012 & 2.40 & $-5.02^{*}$ & $5.99^{*}$ & $10.95^{*}$ & $11.29^{*}$ & $-6.83^{*}$ & $-8.13^{*}$ & $-10.64^{*}$ & 1.57 & 2.37 & 3.13 & 4.73 \\
\hline & 2013 & $13.31^{*}$ & $-18.45^{*}$ & $10.87^{*}$ & $13.73^{*}$ & $21.28^{*}$ & $-12.31^{*}$ & $-12.69^{*}$ & $-15.74^{*}$ & 1.52 & 2.30 & 3.03 & 4.59 \\
\hline & Pooled & $7.85^{*}$ & $-11.74^{*}$ & $8.43^{*}$ & $12.34^{*}$ & $16.29^{*}$ & $-9.57^{*}$ & $-10.41^{*}$ & $-13.19^{*}$ & 1.09 & 1.65 & 2.18 & 3.29 \\
\hline \multirow{3}{*}{$\begin{array}{l}\text { Harvest } \\
\text { duration }\end{array}$} & 2012 & $-5.52^{*}$ & -0.95 & $10.08^{*}$ & 0.48 & 0.38 & -0.78 & $-1.95^{*}$ & -1.75 & 0.98 & 1.48 & 1.95 & 2.95 \\
\hline & 2013 & - & - & - & - & - & - & - & - & - & - & - & - \\
\hline & Pooled & $-2.01^{*}$ & -0.12 & $5.48^{*}$ & 0.36 & -0.22 & -0.59 & $-1.36^{*}$ & $-1.52^{*}$ & 0.62 & 0.94 & 1.24 & 1.88 \\
\hline \multirow[t]{3}{*}{ TSS } & 2012 & $0.21^{*}$ & $0.12^{*}$ & 0.05 & $0.19^{*}$ & -0.08 & $-0.38^{*}$ & -0.10 & -0.01 & 0.06 & 0.09 & 0.12 & 0.17 \\
\hline & 2013 & $0.22^{*}$ & 0.13 & 0.12 & -0.03 & 0.08 & $-0.29^{*}$ & -0.12 & -0.11 & 0.08 & 0.11 & 0.15 & 0.23 \\
\hline & Pooled & $0.22^{*}$ & $0.13^{*}$ & 0.08 & 0.08 & 0.00 & $-0.34^{*}$ & $-0.11^{*}$ & -0.06 & 0.05 & 0.07 & 0.09 & 0.14 \\
\hline \multirow{3}{*}{$\begin{array}{l}\text { Ascorbic } \\
\text { acid }\end{array}$} & 2012 & $1.01^{*}$ & $-0.95^{*}$ & 0.04 & -0.07 & 0.05 & 0.21 & -0.05 & -0.24 & 0.26 & 0.39 & 0.51 & 0.78 \\
\hline & 2013 & -0.36 & $-0.85^{*}$ & $1.30^{*}$ & $1.69^{*}$ & $1.47^{*}$ & $-0.55^{*}$ & $-1.49^{*}$ & $-1.21^{*}$ & 0.26 & 0.40 & 0.53 & 0.80 \\
\hline & Pooled & 0.33 & $-0.90^{*}$ & $0.67^{*}$ & $0.81^{*}$ & $0.76^{*}$ & -0.17 & $-0.77^{*}$ & $-0.73^{*}$ & 0.18 & 0.28 & 0.37 & 0.56 \\
\hline \multirow{3}{*}{$\begin{array}{l}\text { Titrable } \\
\text { acidity }\end{array}$} & 2012 & $-0.05^{*}$ & $-0.06^{*}$ & $0.07^{*}$ & $-0.03^{*}$ & $-0.04^{*}$ & $0.04^{*}$ & $0.03^{*}$ & $0.04^{*}$ & 0.01 & 0.01 & 0.02 & 0.03 \\
\hline & 2013 & $-0.02^{*}$ & $-0.04^{*}$ & 0.01 & $-0.03^{*}$ & $-0.02^{*}$ & 0.01 & $0.04^{*}$ & $0.05^{*}$ & 0.01 & 0.01 & 0.02 & 0.03 \\
\hline & Pooled & $-0.03^{*}$ & $-0.05^{*}$ & $0.04^{*}$ & $-0.03^{*}$ & $-0.03^{*}$ & $0.02^{*}$ & $0.03^{*}$ & $0.04^{*}$ & 0.004 & 0.01 & 0.01 & 0.01 \\
\hline
\end{tabular}

Significant at $5 \%$ level of significance 
Table.4 Estimates of specific combining ability effects for days to 50 per cent flowering, gross yield per plant, marketable yield per plant, total number of fruits per plant and marketable fruits per plant in tomato during 2012, 2013 and pooled over environments under organic conditions

\begin{tabular}{|c|c|c|c|c|c|c|c|c|c|c|c|c|c|c|c|}
\hline \multirow[t]{2}{*}{ Crosses } & \multicolumn{3}{|c|}{ Days to $50 \%$ flowering } & \multicolumn{3}{|c|}{ Gross yield per plant } & \multicolumn{3}{|c|}{$\begin{array}{c}\text { Marketable yield per } \\
\text { plant }\end{array}$} & \multicolumn{3}{|c|}{$\begin{array}{c}\text { Total number of fruits per } \\
\text { plant }\end{array}$} & \multicolumn{3}{|c|}{ Marketable fruits per plant } \\
\hline & 2012 & 2013 & Pooled & 2012 & 2013 & Pooled & & & & & & & & & \\
\hline $\mathbf{P}_{1} \times \mathbf{P}_{2}$ & 0.71 & -1.02 & -0.16 & 0.03 & 0.08 & 0.05 & 0.03 & $0.13 *$ & 0.08 & -2.36 & 0.61 & -0.88 & -1.95 & -0.03 & -0.99 \\
\hline $\mathbf{P}_{1} \times \mathbf{P}_{3}$ & -0.46 & 3.28 & 1.41 & $0.17 *$ & $0.33 *$ & $0.25 *$ & $0.28 *$ & 0.02 & $0.15 *$ & 0.35 & $-10.84 *$ & $-5.25^{*}$ & 0.15 & $-3.59 *$ & -1.72 \\
\hline $\mathbf{P}_{1} \times \mathbf{P}_{4}$ & -0.73 & 1.68 & 0.48 & -0.01 & 0.05 & 0.02 & -0.13 & 0.10 & -0.01 & $-4.98 *$ & 0.09 & $-2.44^{*}$ & -2.25 & 1.26 & -0.50 \\
\hline $\mathbf{P}_{1} \times \mathbf{P}_{5}$ & -1.26 & 1.94 & 0.34 & $0.32 *$ & $0.26^{*}$ & $0.29 *$ & 0.09 & 0.00 & 0.05 & 0.17 & -0.09 & 0.04 & 0.36 & -0.02 & 0.17 \\
\hline $\mathbf{P}_{1} \times \mathbf{P}_{\mathbf{6}}$ & 0.14 & 1.64 & 0.89 & -0.09 & -0.15 & $-0.12 *$ & $-0.23 *$ & 0.01 & $-0.11^{*}$ & $-4.50^{*}$ & -0.21 & $-2.35^{*}$ & $-4.99 *$ & 0.21 & $-2.39 *$ \\
\hline $\mathbf{P}_{1} \times \mathbf{P}_{7}$ & 1.37 & $-4.26^{*}$ & -1.44 & 0.00 & -0.11 & -0.06 & 0.10 & -0.11 & -0.01 & 3.46 & 0.67 & 2.06 & $4.31 *$ & -0.35 & $1.98 *$ \\
\hline $\mathbf{P}_{1} \times \mathbf{P}_{8}$ & -0.73 & -2.29 & -1.51 & -0.07 & -0.17 & $-0.12 *$ & -0.03 & -0.07 & -0.05 & 0.05 & $5.94 *$ & $2.99^{*}$ & 1.48 & 0.58 & 1.03 \\
\hline $\mathbf{P}_{2} \times \mathbf{P}_{3}$ & 0.44 & 1.08 & 0.76 & -0.07 & 0.10 & 0.01 & $0.18^{*}$ & $0.14^{*}$ & $0.16^{*}$ & 0.75 & -0.60 & 0.07 & 1.00 & -0.13 & 0.43 \\
\hline $\mathbf{P}_{2} \times \mathbf{P}_{4}$ & -1.16 & 2.14 & 0.49 & -0.02 & -0.07 & -0.04 & -0.11 & 0.02 & -0.05 & -0.98 & -0.36 & -0.67 & -2.35 & -1.30 & $-1.82^{*}$ \\
\hline $\mathbf{P}_{2} \times \mathbf{P}_{5}$ & -0.69 & -1.92 & -1.31 & -0.09 & -0.08 & -0.08 & 0.12 & -0.09 & 0.01 & $4.52 *$ & $4.05^{*}$ & $4.28 *$ & 2.86 & -1.60 & 0.63 \\
\hline $\mathbf{P}_{2} \times \mathbf{P}_{6}$ & 0.71 & 0.78 & 0.74 & $0.18^{*}$ & $0.18 *$ & $0.18 *$ & 0.02 & 0.10 & 0.06 & 0.43 & $-2.14^{*}$ & -0.85 & -0.60 & 0.51 & -0.05 \\
\hline $\mathbf{P}_{2} \times \mathbf{P}_{7}$ & -0.06 & 1.21 & 0.58 & $0.19 *$ & $0.25 *$ & $0.22 *$ & $0.19 *$ & $0.15 *$ & $0.17 *$ & $8.55^{*}$ & $5.37 *$ & $6.96^{*}$ & $3.00 *$ & $3.60 *$ & $3.30 *$ \\
\hline $\mathbf{P}_{2} \times \mathbf{P}_{8}$ & $-1.83^{*}$ & -2.49 & $-2.16^{*}$ & -0.02 & $0.40^{*}$ & $0.19 *$ & $0.23 *$ & $0.15^{*}$ & $0.19^{*}$ & $4.42 *$ & 0.84 & $2.63^{*}$ & $3.83^{*}$ & 1.44 & $2.64 *$ \\
\hline $\mathbf{P}_{3} \times \mathbf{P}_{4}$ & -1.33 & -1.56 & -1.44 & 0.11 & $0.29 *$ & $0.20 *$ & 0.02 & 0.11 & 0.07 & 3.49 & $5.12 *$ & $4.30^{*}$ & 1.76 & $3.60 *$ & $2.68^{*}$ \\
\hline $\mathbf{P}_{3} \times \mathbf{P}_{5}$ & $1.81 *$ & -2.96 & -0.57 & $0.41^{*}$ & -0.09 & $0.16^{*}$ & $0.19^{*}$ & $0.21 *$ & $0.20 *$ & 2.85 & $-2.81 *$ & 0.02 & 2.71 & $4.28^{*}$ & $3.50^{*}$ \\
\hline $\mathbf{P}_{3} \times \mathbf{P}_{\mathbf{6}}$ & $-2.13 *$ & 1.74 & -0.19 & $-0.16^{*}$ & 0.14 & -0.01 & 0.00 & $-0.38 *$ & $-0.19 *$ & $4.47 *$ & $6.31 *$ & $5.39 *$ & 2.90 & $-6.34 *$ & -1.72 \\
\hline $\mathbf{P}_{3} \times \mathbf{P}_{7}$ & -0.89 & 0.84 & -0.02 & $0.20 *$ & $0.71 *$ & $0.46^{*}$ & $-0.19^{*}$ & 0.03 & -0.08 & $-12.10^{*}$ & -0.04 & $-6.07 *$ & $-3.62^{*}$ & -0.11 & $-1.87 *$ \\
\hline $\mathbf{P}_{3} \times \mathbf{P}_{8}$ & -0.66 & 0.81 & 0.08 & -0.02 & $0.43^{*}$ & $0.21^{*}$ & $-0.26^{*}$ & $-0.20^{*}$ & $-0.23^{*}$ & $-7.68 *$ & $2.32 *$ & $-2.68^{*}$ & $-5.41^{*}$ & $-5.65^{*}$ & $-5.53^{*}$ \\
\hline $\mathbf{P}_{4} \times \mathbf{P}_{5}$ & $-1.79 *$ & -1.56 & -1.67 & $0.19 *$ & -0.02 & 0.08 & $0.38^{*}$ & $0.18^{*}$ & $0.28^{*}$ & -0.59 & 0.10 & -0.24 & 2.63 & 1.34 & $1.99 *$ \\
\hline $\mathbf{P}_{4} \times \mathbf{P}_{6}$ & $1.61 *$ & -1.52 & 0.04 & $0.34^{*}$ & $0.65^{*}$ & $0.49 *$ & $0.45^{*}$ & $0.21 *$ & $0.33 *$ & $4.33^{*}$ & 1.27 & $2.80^{*}$ & $7.23^{*}$ & 0.71 & $3.97 *$ \\
\hline $\mathbf{P}_{4} \times \mathbf{P}_{7}$ & $-3.83 *$ & $-4.42 *$ & $-4.12 *$ & $0.30 *$ & $0.36^{*}$ & $0.33 *$ & $0.23 *$ & 0.09 & $0.16^{*}$ & $6.47 *$ & 0.84 & $3.65^{*}$ & $4.10 *$ & 0.88 & $2.49 *$ \\
\hline $\mathbf{P}_{4} \times \mathbf{P}_{8}$ & $-1.59 *$ & 2.21 & 0.31 & $0.17 *$ & $0.43^{*}$ & $0.30 *$ & 0.14 & -0.02 & 0.06 & $5.64 *$ & $-2.71 *$ & 1.47 & $3.52 *$ & $-2.02 *$ & 0.75 \\
\hline $\mathbf{P}_{5} \times \mathbf{P}_{6}$ & -0.59 & -1.92 & -1.26 & 0.12 & $0.41^{*}$ & $0.26^{*}$ & $0.35^{*}$ & $0.26^{*}$ & $0.30 *$ & $9.50 *$ & $-3.24 *$ & $3.13^{*}$ & $10.65^{*}$ & 1.95 & $6.30^{*}$ \\
\hline $\mathbf{P}_{5} \times \mathbf{P}_{7}$ & -1.03 & 1.18 & 0.08 & -0.11 & 0.03 & -0.04 & $-0.17 *$ & 0.10 & -0.04 & -2.94 & $2.24^{*}$ & -0.35 & $-4.12 *$ & 1.45 & -1.34 \\
\hline $\mathbf{P}_{5} \times \mathbf{P}_{8}$ & -1.13 & 2.48 & 0.68 & 0.03 & 0.15 & 0.09 & 0.00 & $0.27 *$ & $0.13 *$ & 1.61 & $6.44 *$ & $4.02 *$ & 0.38 & $5.75^{*}$ & $3.07 *$ \\
\hline $\mathbf{P}_{6} \times \mathbf{P}_{7}$ & 0.04 & 0.54 & 0.29 & -0.06 & $-0.26^{*}$ & $-0.16^{*}$ & -0.01 & $0.15 *$ & 0.07 & -2.14 & 0.91 & -0.61 & -2.87 & $3.48 *$ & 0.31 \\
\hline $\mathbf{P}_{6} \times \mathbf{P}_{8}$ & -1.39 & 1.84 & 0.23 & $0.25^{*}$ & $-0.40^{*}$ & -0.07 & 0.03 & -0.08 & -0.03 & -2.80 & $-2.25^{*}$ & $-2.52^{*}$ & -2.58 & -1.15 & $-1.86^{*}$ \\
\hline $\mathbf{P}_{7} \times \mathbf{P}_{8}$ & 0.51 & 0.94 & 0.73 & $0.19^{*}$ & $-0.27 *$ & -0.04 & $0.24 *$ & 0.01 & $0.13^{*}$ & 2.66 & 0.63 & 1.64 & 2.15 & -1.18 & 0.48 \\
\hline $\mathrm{SE}(\mathrm{Sij}) \pm$ & 0.77 & 1.75 & 0.95 & 0.08 & 0.09 & 0.06 & 0.07 & 0.07 & 0.05 & 2.06 & 1.02 & 1.15 & 1.47 & 1.01 & 0.89 \\
\hline $\mathrm{SE}(\mathrm{Sij}-\mathrm{Sik}) \pm$ & 1.13 & 2.58 & 1.41 & 0.12 & 0.13 & 0.09 & 0.11 & 0.10 & 0.07 & 3.05 & 1.50 & 1.70 & 2.17 & 1.49 & 1.32 \\
\hline SE $($ Sij-Skl $) \pm$ & 1.07 & 2.44 & 1.33 & 0.11 & 0.12 & 0.08 & 0.10 & 0.09 & 0.07 & 2.87 & 1.42 & 1.60 & 2.05 & 1.41 & 1.24 \\
\hline $\mathrm{CD}(\mathrm{Sij})$ & 1.53 & 3.48 & 1.90 & 0.16 & 0.18 & 0.12 & 0.15 & 0.13 & 0.10 & 4.11 & 2.02 & 2.29 & 2.93 & 2.01 & 1.78 \\
\hline CD(Sij-Sik) & 2.26 & 5.15 & 2.81 & 0.23 & 0.26 & 0.18 & 0.22 & 0.20 & 0.15 & 6.07 & 2.99 & 3.39 & 4.33 & 2.98 & 2.63 \\
\hline CD (Sij-Skl) & 2.13 & 4.86 & 2.65 & 0.22 & 0.25 & 0.17 & 0.21 & 0.19 & 0.14 & 5.73 & 2.82 & 3.19 & 4.08 & 2.81 & 2.48 \\
\hline
\end{tabular}

*Significant at $5 \%$ level of significance 
Table.5 Estimates of specific combining ability effects fruit weight, fruit shape index, pericarp thickness, locules per fruit and plant height in tomato during 2012, 2013 and pooled over environments under organic conditions

\begin{tabular}{|c|c|c|c|c|c|c|c|c|c|c|c|c|c|c|c|}
\hline \multirow[t]{2}{*}{ Crosses } & \multicolumn{3}{|c|}{ Fruit weight } & \multicolumn{3}{|c|}{ Fruit shape index } & \multicolumn{3}{|c|}{ Pericarp thickness } & \multicolumn{3}{|c|}{ Locules per fruit } & \multicolumn{3}{|c|}{ Plant height } \\
\hline & 2012 & 2013 & Pooled & & & & & & & & & & & & \\
\hline $\mathbf{P}_{1} \times \mathbf{P}_{2}$ & $6.66 *$ & $5.72 *$ & $6.19 *$ & - & - & $-0.06^{*}$ & -0.24 & -0.39 & $-0.31 *$ & 0.31 & $0.33 *$ & $0.32 *$ & - & $9.43 *$ & 6.60 \\
\hline $\mathbf{P}_{1} \times \mathbf{P}_{3}$ & $6.96 *$ & $6.91 *$ & $6.93 *$ & - & - & -0.01 & 0.17 & 0.33 & 0.25 & 0.14 & 0.01 & 0.08 & - & -7.60 & -2.91 \\
\hline $\mathbf{P}_{1} \times \mathbf{P}_{4}$ & 1.18 & 0.82 & 1.00 & - & - & $-0.05^{*}$ & -0.03 & 0.29 & 0.13 & 0.19 & 0.13 & 0.16 & - & 1.75 & 0.03 \\
\hline$P_{1} \times P_{5}$ & 1.91 & 1.70 & 1.81 & - & - & -0.03 & 0.35 & -0.01 & 0.17 & -0.08 & $-0.37 *$ & -0.22 & - & 5.14 & 3.87 \\
\hline $\mathbf{P}_{1} \times \mathbf{P}_{6}$ & 0.83 & 2.22 & 1.52 & - & - & 0.01 & 0.10 & -0.26 & -0.08 & -0.06 & $0.74 *$ & $0.34 *$ & - & -1.51 & 0.24 \\
\hline $\mathbf{P}_{1} \times \mathbf{P}_{7}$ & $-4.62 *$ & $-4.85^{*}$ & $-4.73 *$ & - & - & 0.00 & -0.09 & 0.08 & -0.01 & $-0.42 *$ & $-0.31 *$ & $-0.36^{*}$ & - & 2.44 & 1.98 \\
\hline $\mathbf{P}_{1} \times \mathbf{P}_{8}$ & $-3.49 *$ & $-4.92 *$ & $-4.21 *$ & - & - & -0.01 & 0.32 & 0.31 & $0.32 *$ & -0.37 & $-0.38 *$ & $-0.37 *$ & - & 5.66 & 5.93 \\
\hline $\mathbf{P}_{2} \times \mathbf{P}_{3}$ & $6.10 *$ & $4.50 *$ & $5.30 *$ & - & - & -0.02 & -0.16 & -0.27 & -0.22 & -0.11 & 0.02 & -0.05 & - & -4.39 & 1.30 \\
\hline $\mathbf{P}_{2} \times \mathbf{P}_{4}$ & 1.03 & 1.49 & 1.26 & - & - & 0.00 & $0.51 *$ & $0.49 *$ & $0.50 *$ & -0.06 & 0.00 & -0.03 & - & $-21.58 *$ & $-10.25^{*}$ \\
\hline $\mathbf{P}_{2} \times \mathbf{P}_{5}$ & -1.45 & -0.74 & -1.09 & - & - & 0.04 & 0.10 & $-0.42 *$ & -0.16 & $-0.43^{*}$ & -0.02 & -0.23 & - & $-26.81 *$ & $-14.31 *$ \\
\hline $\mathbf{P}_{2} \times \mathbf{P}_{6}$ & 0.40 & 2.48 & 1.44 & - & - & 0.03 & -0.12 & -0.13 & -0.13 & -0.35 & $-0.38 *$ & $-0.37 *$ & - & -6.41 & -4.35 \\
\hline $\mathbf{P}_{2} \times \mathbf{P}_{7}$ & 1.90 & -0.74 & 0.58 & - & - & 0.00 & -0.32 & -0.19 & -0.26 & 0.00 & -0.04 & -0.02 & - & -1.03 & -2.18 \\
\hline $\mathbf{P}_{2} \times \mathbf{P}_{8}$ & 2.45 & 2.56 & 2.50 & - & - & -0.02 & 0.06 & 0.23 & 0.15 & 0.15 & -0.21 & -0.03 & - & -5.18 & -1.71 \\
\hline $\mathbf{P}_{3} \times \mathbf{P}_{4}$ & -2.44 & $-4.40 *$ & $-3.42 *$ & - & - & -0.04 & $0.73 *$ & $0.47 *$ & $0.60 *$ & 0.31 & $0.34 *$ & $0.33 *$ & - & $-10.57 *$ & $-7.86^{*}$ \\
\hline $\mathbf{P}_{3} \times \mathbf{P}_{5}$ & 0.59 & 1.62 & 1.10 & - & - & -0.01 & -0.32 & -0.17 & -0.24 & $0.44 *$ & $0.45^{*}$ & $0.45 *$ & - & $-19.54 *$ & $-11.90 *$ \\
\hline $\mathbf{P}_{3} \times \mathbf{P}_{6}$ & $-7.06^{*}$ & $-6.59 *$ & $-6.83 *$ & - & - & $-0.05^{*}$ & $0.70 *$ & $0.45^{*}$ & $0.58 *$ & -0.04 & $-0.38^{*}$ & -0.21 & - & $20.07 *$ & $13.27 *$ \\
\hline $\mathbf{P}_{\mathbf{3}} \times \mathbf{P}_{7}$ & -3.16 & 0.58 & -1.29 & - & - & $0.05 *$ & -0.35 & $-0.41 *$ & $-0.38^{*}$ & $-0.40 *$ & $-0.50 *$ & $-0.45^{*}$ & - & $22.38^{*}$ & $12.68 *$ \\
\hline $\mathbf{P}_{3} \times \mathbf{P}_{8}$ & -2.09 & 1.46 & -0.32 & - & - & -0.02 & 0.01 & 0.02 & 0.02 & -0.27 & $-0.40 *$ & $-0.34 *$ & - & $15.97 *$ & $8.78 *$ \\
\hline $\mathbf{P}_{4} \times \mathbf{P}_{5}$ & $7.52 *$ & 3.38 & $5.45^{*}$ & - & - & -0.03 & 0.04 & $0.46^{*}$ & 0.25 & 0.19 & $0.50 *$ & $0.35^{*}$ & - & 3.22 & 1.59 \\
\hline $\mathbf{P}_{4} \times \mathbf{P}_{6}$ & 1.03 & $7.16^{*}$ & $4.09 *$ & - & - & 0.01 & 0.23 & 0.35 & 0.29 & $-0.49^{*}$ & $-0.72 *$ & $-0.60 *$ & - & 8.21 & $6.99 *$ \\
\hline $\mathbf{P}_{4} \times \mathbf{P}_{7}$ & 1.52 & 1.19 & 1.35 & - & - & $0.06^{*}$ & $0.73 *$ & $0.62 *$ & $0.68 *$ & -0.30 & $-0.51 *$ & $-0.40 *$ & - & $10.86^{*}$ & $8.29 *$ \\
\hline $\mathbf{P}_{4} \times \mathbf{P}_{8}$ & -0.88 & 2.57 & 0.84 & - & - & -0.02 & 0.28 & 0.18 & 0.23 & -0.32 & -0.15 & -0.23 & - & $14.18^{*}$ & $9.36^{*}$ \\
\hline $\mathbf{P}_{5} \times \mathbf{P}_{6}$ & 3.22 & $7.57 *$ & $5.39 *$ & - & - & -0.01 & 0.33 & 0.37 & $0.35 *$ & $-0.40 *$ & -0.22 & $-0.31 *$ & - & $17.52 *$ & $13.80 *$ \\
\hline $\mathbf{P}_{5} \times \mathbf{P}_{7}$ & 1.95 & 2.15 & 2.05 & - & - & -0.04 & 0.36 & 0.38 & $0.37 *$ & 0.20 & 0.06 & 0.13 & - & $13.90 *$ & $12.79 *$ \\
\hline $\mathbf{P}_{5} \times \mathbf{P}_{8}$ & -2.04 & -0.44 & -1.24 & - & - & 0.00 & $-0.46^{*}$ & $-0.53 *$ & $-0.49 *$ & -0.37 & $-0.44 *$ & $-0.40 *$ & - & $25.75^{*}$ & $17.50 *$ \\
\hline $\mathbf{P}_{6} \times \mathbf{P}_{7}$ & $5.87 *$ & -0.44 & 2.72 & - & - & -0.02 & 0.21 & 0.13 & 0.17 & -0.23 & -0.16 & -0.20 & - & -2.40 & -2.61 \\
\hline $\mathbf{P}_{6} \times \mathbf{P}_{8}$ & $7.04 *$ & -0.14 & $3.45 *$ & - & - & $0.06^{*}$ & 0.20 & 0.03 & 0.11 & 0.18 & $0.40 *$ & $0.29 *$ & - & $-10.32 *$ & $-7.41 *$ \\
\hline $\mathbf{P}_{7} \times \mathbf{P}_{8}$ & $6.41 *$ & 4.12 & $5.26 *$ & - & - & $-0.06^{*}$ & -0.03 & 0.03 & 0.00 & $1.05^{*}$ & $0.95 *$ & $1.00 *$ & - & $-17.39 *$ & $-13.35^{*}$ \\
\hline $\mathrm{SE}(\mathrm{Sij}) \pm$ & 1.75 & 2.17 & 1.39 & - & - & 0.02 & 0.22 & 0.20 & 0.15 & 0.20 & 0.14 & 0.12 & - & 4.67 & 3.35 \\
\hline SE(Sij-Sik $) \pm$ & 2.59 & 3.20 & 2.06 & - & - & 0.03 & 0.33 & 0.30 & 0.22 & 0.29 & 0.21 & 0.18 & - & 6.90 & 4.96 \\
\hline SE (Sij-Skl) \pm & 2.44 & 3.02 & 1.94 & - & - & 0.03 & 0.31 & 0.28 & 0.21 & 0.27 & 0.20 & 0.17 & - & 6.51 & 4.68 \\
\hline $\mathrm{CD}(\mathrm{Sij})$ & 3.49 & 4.32 & 2.78 & - & - & 0.05 & 0.44 & 0.40 & 0.30 & 0.39 & 0.29 & 0.24 & - & 9.30 & 6.68 \\
\hline CD(Sij-Sik) & 5.16 & 6.39 & 4.11 & - & - & 0.07 & 0.66 & 0.59 & 0.44 & 0.58 & 0.43 & 0.36 & - & 13.76 & 9.88 \\
\hline CD (Sij-Skl) & 4.87 & 6.02 & 3.87 & - & - & 0.06 & 0.62 & 0.55 & 0.42 & 0.54 & 0.40 & 0.34 & - & 12.97 & 9.32 \\
\hline
\end{tabular}

*Significant at $5 \%$ level of significance 
Table.6 Estimates of specific combining ability effects for harvest duration, total soluble solids, ascorbic acid and titrable acidity flowering in tomato during 2012, 2013 and pooled over environments under organic conditions

\begin{tabular}{|c|c|c|c|c|c|c|c|c|c|c|c|c|}
\hline \multirow{2}{*}{$\begin{array}{l}\text { Crosses } \\
\mathbf{P}_{1} \times \mathbf{P}_{2}\end{array}$} & \multicolumn{3}{|c|}{ Harvest duration } & \multicolumn{3}{|c|}{ Total soluble solids } & \multicolumn{3}{|c|}{ Ascorbic acid } & \multicolumn{3}{|c|}{ Titrable acidity } \\
\hline & $6.26 *$ & $-5.35^{*}$ & 0.46 & -0.13 & - & $-0.32 *$ & $6.32 *$ & $3.51 *$ & $4.92 *$ & 0.03 & 0.01 & $0.02 *$ \\
\hline $\mathbf{P}_{1} \times \mathbf{P}_{3}$ & -0.10 & $8.15^{*}$ & $4.02 *$ & $0.41 *$ & - & $0.39 *$ & $-4.60 *$ & 0.64 & $-1.98 *$ & 0.01 & -0.03 & -0.01 \\
\hline $\mathbf{P}_{1} \times \mathbf{P}_{4}$ & $8.16^{*}$ & -4.21 & 1.97 & -0.20 & - & -0.04 & -0.53 & -1.48 & -1.01 & $0.08 *$ & $0.10 *$ & $0.09 *$ \\
\hline $\mathbf{P}_{1} \times \mathbf{P}_{5}$ & 3.60 & $-10.81 *$ & -3.61 & $0.41 *$ & - & 0.28 & $2.13 *$ & -1.29 & 0.42 & 0.03 & 0.05 & $0.04 *$ \\
\hline $\mathbf{P}_{1} \times \mathbf{P}_{6}$ & -4.24 & 1.75 & -1.24 & -0.06 & - & -0.07 & $5.36^{*}$ & 1.45 & $3.40 *$ & -0.03 & $0.07 *$ & $0.02 *$ \\
\hline $\mathbf{P}_{1} \times \mathbf{P}_{7}$ & $-7.07 *$ & $7.12 *$ & 0.02 & $-0.45^{*}$ & - & $-0.42 *$ & $-2.97 *$ & $-4.68 *$ & $-3.82 *$ & -0.05 & $0.07 *$ & 0.01 \\
\hline $\mathbf{P}_{1} \times \mathbf{P}_{8}$ & -0.94 & $-5.01 *$ & -2.98 & -0.20 & - & 0.00 & $-8.15^{*}$ & $2.65^{*}$ & $-2.75^{*}$ & $-0.07 *$ & 0.01 & $-0.03 *$ \\
\hline $\mathbf{P}_{2} \times \mathbf{P}_{3}$ & 0.00 & $-6.05^{*}$ & -3.03 & -0.04 & - & 0.01 & 1.55 & $3.74 *$ & $2.65^{*}$ & -0.04 & 0.04 & 0.00 \\
\hline $\mathbf{P}_{2} \times \mathbf{P}_{4}$ & 2.60 & 0.25 & 1.42 & -0.21 & - & -0.15 & $-4.65^{*}$ & 0.99 & $-1.83^{*}$ & $0.06^{*}$ & $0.11 *$ & $0.08 *$ \\
\hline $\mathbf{P}_{2} \times \mathbf{P}_{5}$ & $9.03 *$ & 0.65 & $4.84 *$ & 0.10 & - & 0.13 & -0.85 & 1.02 & 0.08 & $0.06^{*}$ & 0.01 & $0.04 *$ \\
\hline $\mathbf{P}_{2} \times \mathbf{P}_{6}$ & $-6.14 *$ & 4.55 & -0.79 & 0.26 & - & $0.30 *$ & $-4.61 *$ & $-4.21 *$ & $-4.41 *$ & -0.03 & -0.02 & $-0.03 *$ \\
\hline $\mathbf{P}_{2} \times \mathbf{P}_{7}$ & 5.70 & -3.75 & 0.97 & -0.22 & - & -0.16 & $-1.58 *$ & $-5.97 *$ & $-3.77 *$ & 0.00 & 0.05 & $0.03 *$ \\
\hline $\mathbf{P}_{2} \times \mathbf{P}_{8}$ & -1.50 & 3.45 & 0.97 & 0.19 & - & -0.03 & $3.72 *$ & 1.54 & $2.63 *$ & $-0.07 *$ & -0.03 & $-0.05^{*}$ \\
\hline $\mathbf{P}_{3} \times \mathbf{P}_{4}$ & 0.56 & -2.91 & -1.18 & 0.30 & - & 0.19 & $2.15^{*}$ & $-2.19 *$ & -0.02 & 0.03 & 0.03 & $0.03 *$ \\
\hline $\mathbf{P}_{3} \times \mathbf{P}_{5}$ & 1.66 & 0.82 & 1.24 & -0.16 & - & -0.11 & 1.44 & -0.20 & 0.62 & $0.07 *$ & 0.03 & $0.05 *$ \\
\hline $\mathbf{P}_{3} \times \mathbf{P}_{6}$ & 2.50 & 1.39 & 1.94 & -0.13 & - & -0.26 & 0.06 & $3.98 *$ & $2.02 *$ & 0.05 & -0.05 & 0.00 \\
\hline $\mathbf{P}_{3} \times \mathbf{P}_{7}$ & 2.66 & $-8.25 *$ & -2.79 & $0.38 *$ & - & $0.45^{*}$ & $3.03 *$ & 1.49 & $2.26^{*}$ & $0.09 *$ & $0.06^{*}$ & $0.07 *$ \\
\hline $\mathbf{P}_{3} \times \mathbf{P}_{8}$ & $9.46 *$ & 3.95 & $6.71 *$ & 0.23 & - & 0.13 & 0.94 & $-3.59 *$ & $-1.32 *$ & $0.07 *$ & $0.08 *$ & $0.07 *$ \\
\hline $\mathbf{P}_{4} \times \mathbf{P}_{5}$ & -0.74 & 3.79 & 1.52 & 0.00 & - & -0.11 & 0.56 & $-6.07 *$ & $-2.76^{*}$ & 0.00 & $0.06^{*}$ & $0.03 *$ \\
\hline $\mathbf{P}_{4} \times \mathbf{P}_{6}$ & -0.90 & -2.65 & -1.78 & $-0.40^{*}$ & - & -0.09 & $2.15 *$ & -0.37 & 0.89 & $-0.08 *$ & -0.01 & $-0.05^{*}$ \\
\hline $\mathbf{P}_{4} \times \mathbf{P}_{7}$ & $9.26 *$ & $5.05^{*}$ & $7.16^{*}$ & 0.11 & - & 0.05 & 0.32 & 0.32 & 0.32 & $-0.09 *$ & 0.01 & $-0.04 *$ \\
\hline $\mathbf{P}_{4} \times \mathbf{P}_{8}$ & 4.73 & $-6.75^{*}$ & -1.01 & -0.24 & - & -0.18 & $3.81 *$ & $3.68^{*}$ & $3.74 *$ & $0.08 *$ & -0.03 & $0.03 *$ \\
\hline $\mathbf{P}_{5} \times \mathbf{P}_{6}$ & 4.86 & 2.42 & 3.64 & -0.23 & - & -0.09 & 1.16 & $-2.93 *$ & -0.88 & 0.00 & 0.02 & 0.01 \\
\hline $\mathbf{P}_{5} \times \mathbf{P}_{7}$ & 4.70 & 2.12 & 3.41 & 0.12 & - & 0.03 & 1.52 & $5.81^{*}$ & $3.67 *$ & -0.04 & $0.07 *$ & $0.02 *$ \\
\hline $\mathbf{P}_{5} \times \mathbf{P}_{8}$ & 2.16 & -4.68 & -1.26 & $0.43^{*}$ & - & $0.37 *$ & 0.66 & -1.37 & -0.36 & $-0.07 *$ & 0.05 & -0.01 \\
\hline $\mathbf{P}_{6} \times \mathbf{P}_{7}$ & 4.86 & -1.65 & 1.61 & 0.28 & - & $0.30 *$ & $3.99 *$ & $2.73 *$ & $3.36 *$ & $0.16^{*}$ & $0.07 *$ & $0.11 *$ \\
\hline $\mathbf{P}_{6} \times \mathbf{P}_{8}$ & $8.33^{*}$ & -0.45 & $3.94 *$ & 0.07 & - & 0.12 & -0.20 & $-2.65^{*}$ & $-1.43^{*}$ & $0.13^{*}$ & $0.10 *$ & $0.11 *$ \\
\hline $\mathbf{P}_{7} \times \mathbf{P}_{8}$ & $-6.84 *$ & -2.08 & $-4.46^{*}$ & $0.58^{*}$ & - & $0.46^{*}$ & $2.59 *$ & 0.75 & $1.67 *$ & $0.12 *$ & 0.02 & $0.07 *$ \\
\hline SE(Sij) \pm & 3.00 & 2.38 & 1.91 & 0.18 & - & 0.15 & 0.79 & 0.81 & 0.57 & 0.03 & 0.03 & 0.01 \\
\hline $\mathrm{SE}($ Sij-Sik $) \pm$ & 4.43 & 3.52 & 2.83 & 0.26 & - & 0.22 & 1.17 & 1.20 & 0.84 & 0.04 & 0.04 & 0.02 \\
\hline SE (Sij-Skl) \pm & 4.18 & 3.32 & 2.67 & 0.25 & - & 0.20 & 1.10 & 1.13 & 0.79 & 0.04 & 0.04 & 0.02 \\
\hline $\mathrm{CD}(\mathrm{Sij})$ & 5.97 & 4.74 & 3.81 & 0.35 & - & 0.29 & 1.57 & 1.61 & 1.13 & 0.06 & 0.06 & 0.02 \\
\hline CD(Sij-Sik) & 8.84 & 7.02 & 5.64 & 0.52 & - & 0.43 & 2.33 & 2.39 & 1.67 & 0.09 & 0.09 & 0.04 \\
\hline CD (Sij-Skl) & 8.33 & 6.62 & 5.32 & 0.49 & - & 0.40 & 2.19 & 2.25 & 1.57 & 0.08 & 0.08 & 0.03 \\
\hline
\end{tabular}

*Significant at $5 \%$ level of significance 
The best five specific combinations for marketable yield per plant in pooled environment were $\mathrm{P}_{4} \times \mathrm{P}_{6}, \mathrm{P}_{5} \times \mathrm{P}_{6}, \mathrm{P}_{4} \times \mathrm{P}_{5}$, $\mathrm{P}_{3} \times \mathrm{P}_{5}$ and $\mathrm{P}_{2} \times \mathrm{P}_{8}$. The cross combination $\mathrm{P}_{4} \times \mathrm{P}_{6} \quad($ Palam Pride $\times$ BWR-5) also revealed significant desirable SCA effects in component traits viz., gross yield per plant, total number of fruits per plant, marketable fruits per plant, fruit weight and plant height. The cross combination $\mathrm{P}_{5} \times \mathrm{P}_{6}$ exhibited desirable SCA effects for gross yield per plant, total number of fruits per plant, marketable fruits per plant, fruit weight, pericarp thickness and plant height, whereas $\mathrm{P}_{4} \times \mathrm{P}_{5}$ for marketable fruits per plant, fruit weight, locules per fruit and titrable acidity. On the basis of specific combining ability effects, it can be concluded that among 28 crosscombinations studied, no single crosscombination possessed consistently significant SCA effects for all the traits studied.

\section{References}

A.O.A.C. 1970. Official Methods of Analysis of the Association of Official Analytical Chemists. (W Horwitz, ed). Benjamin Franklin Station, Washington, D.C.

Ahmad, S., A.K.M. Quamruzzaman and M.N. Uddin. 2009. Combining ability estimates of tomato (Solanum lycopersicum) in late summer. SAARC Journal of Agriculture 7: 43-56

Chishti, S.A.S., A.A. Khan, S. Bushra and I.A. Khan. 2008. Analysis of combining ability for yield, yield components and quality characters in tomato (Lycopersicon esculentum Mill.). Journal of Agricultural Research 46: 325-332

Dhaliwal, M.S. and Cheema, D.S. 2011. Genetic analysis of fruit weight and total yield in tomato and development of $F_{1}$ hybrids for cultivation under leaf curl virus infested conditions. Crop Improvement 38: 60-66

Farzane, A., H. Nemati, H. Arouiee and A.M. Kakhki. 2013. The estimate of heterosis and combining ability of some morphological characters in tomato transplants (Lycopersicon esculentum M.). International Journal of Farming and Allied Sciences 2: 290-295

Griffing, B. 1956. Concept of general and specific combining ability in relation to diallel crossing system. Australian Journal of Biological Science 9: 463493

Joshi, A., M.C. Thakur and U.K. Kohli. 2005. Heterosis and combining ability for shelf life, whole fruit firmness and related traits in tomato. Indian Journal of Horticulture 62: 33-36

Kumar, R., K. Srivastava, N.P. Singh, N.K. Vasistha, R.K. Singh and M.K. Singh. 2013. Combining ability analysis for yield and quality traits in tomato (Solanum lycopersicum L.). Journal of Agricultural Science 5: 213-218

Pandey, S.K., J.Dixit, V.N. Pathak and P.K. Singh. 2006. Line $\times$ Tester analysis for yield and quality characters in tomato. Vegetable Science 33: 13-17

Ranganna, S. 1979. Manuals of Analysis of Fruits and Vegetable Products. Tata McGraw Hill Book Company, New Delhi

Saleem M.Y., M. Asghar, Q. Iqbal, A.U. Rahman and M. Akram. 2013. Diallel analysis of yield and some yield components in tomato (Solanum lycopersicum L.). Pakistan Journal of Botany 45: 1247-1250

Shankar, A., R.V.S.K. Reddy, M. Sujatha and M. Pratap. 2013. Combining ability and gene action studies for yield and yield contributing traits in 
tomato (Solanum lycopersicum L.). Helix 6: 431-435

Sharma, D. and Sharma, H.R. 2010. Combining ability analysis for yield and other horticultural traits in tomato. Indian Journal of Horticulture 67: 402-405

Sharma, P., Vidyasagar and N.Bhardwaj. 2007. Combining ability in bacterial wilt resistant genotypes of tomato. Environment and Ecology 25: 196-200

Singh, A.K. and Asati, B.S. 2011. Combining ability and heterosis studies in tomato under bacterial wilt condition. Bangladesh Journal of Agricultural Research. 36: 313-318

Singh, B., S. Kaul, D. Kumar and V.Kumar. 2010. Combining ability for yield and its contributing characters in tomato. Indian Journal of Horticulture 67: 5055

Yadav, S.K., B.K. Singh, D.K. Baranwal and S.S. Solankey. 2013. Genetic study of heterosis for yield and quality components in tomato (Solanum lycopersicum). African Journal of Agricultural Research 8: 5585-5591.

\section{How to cite this article:}

Nisha Thakur, Sanjay Chadha and Mayanglambam Bilashini Devi. 2019. Organic Tomatoes: Combining Ability for fruit yield and Component Traits in Tomato (Solanum lycopersicum L.) under Mid Himalayan Region. Int.J.Curr.Microbiol.App.Sci. 8(01): 2099-2112. doi: https://doi.org/10.20546/ijcmas.2019.801.220 